\title{
Criteri di semifattorialità e di fattorialità per gli anelli locali con applicazioni geometriche.
}

\author{
L. BĂDescu (Bucarest) - M. FioRentini (Ferrara) (*)
}

A Beniamino Segre per il suo $70^{\circ}$ compleanno

Summary. - We give two criteria of almost factoriality and factoriality for lacal rings. Two geometrical applications follow.

\section{Introduzione.}

In questa Nota si danno due criteri di semifattorialità (fattorialità) per gli anelli locali (il Teorema A con i Corollari 1, 2 e 3 e il Teorema B).

Il Teorema A si ispira allo studio delle contrazioni razionali (cfr. [1]) ed ha come applicazione geometrica la semifattorialità (fattorialità) degli anelli locali che compaiono in una contrazione razionale (Teorema A bis).

I1 Teorema B invece si ricollega alle contrazioni ad un punto di una varietà intersezione completa (efr. [2]) ed ha come applicazione geometrica la semifattorialità (fattorialità) dell'anello locale di un tale punto (Teorema $\mathrm{B}$ bis).

Notazioni e terminologia sono desunti da [4] e [5]; più precisamente, si è impiegato il linguaggio degli schemi, in ipotesi che consentono l'identificazione al caso della Geometria algebrica classica: infatti i $K$-schemi separati, integri e di tipo finito sopra un campo algebricamente chiuso $K$, hanno come corrispondenti le varietà algebriche irriducibili.

\section{1. - Definizioni e richiami.}

Definizione 1. - Si dice che un morfismo di schemi $f: Y \rightarrow X$ è una immersione chiusa se esso si può fattorizzare mediante il diagramma

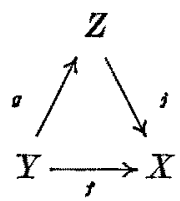

(*) Entrata in Redazione il 12 gennaio 1974. 
dove $g$ è un isomorfismo, $Z$ è un sottoschema chiuso di $X$ e $j$ è il morfismo d'iniezione canonica.

Sia ora $j: Y^{r} \rightarrow Y$ una immersione chiusa di schemi integri localmente noetheriani di ideale $J$ nel fascio strutturale $O_{Y}, f: X \rightarrow Y$ la dilatazione di $Y$ di centro $Y^{\prime}$, $X^{\prime}=f^{-1}\left(Y^{\prime}\right), i: X^{\prime} \rightarrow X$ l'immersione corrispondente d'ideale $I=O_{X}(1)$. Sia $Y$ normale in ogni punto di $Y^{\prime}$.

DefINIZIONe 2. - Nelle ipotesi precedenti, la immersione chiusa $j: Y^{\prime} \rightarrow Y$ dicesi immersione razionale, se una delle condizioni equivalenti è soddisfatta:

(i) se

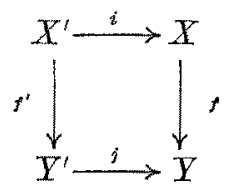

è il diagramma cartesiano della dilatazione di $Y$ di centro $Y^{\prime}$, allora esiste un fascio localmente libero $E$ di rango $r \geqslant 2$ su $T^{\prime}$ tale che $X^{\prime}=P(E)$ (il fibrato proiettivo associato al fascio $E)$ e $i^{*} I=O_{P(E)}(s), s>0$;

(ii) esiste un fascio $E$ localmente libero di rango $r \geqslant 2$ su $Y^{\prime}$ tale che, se $\mathbb{S}(E)$ è l'algebra simmetrica di $E$, vi è un isomorfismo di algebre graduate

$$
\alpha: \underset{n \geqslant 0}{\oplus} J^{n} / J^{n+1} \rightarrow S(E)^{(s)}
$$

con $s>0$, dove si è denotata con $S(E)^{(s)}$, vedi [4], ch. II, $\$ 2$, la sottoalgebra di $S(E)$ tale che $\left(S(E)^{(s)}\right)_{n}=S(E)_{n s}=S^{n s}(E)$.

La implicazione (i) $\Rightarrow$ (ii) segue dal coroll. 1 del teor. 1 di [1]. La implicazione (ii) $\Rightarrow$ (i) segue da [4], ch. II.

OSgERVAZrone 1. - Se $j: Y^{\prime} \rightarrow Y$ è una immersione razionale, dalla (ii) della def. 2 segue che $J / J^{2}$ ̀̀ localmente libero di rango $\left(\begin{array}{c}r+s-1 \\ r-1\end{array}\right)$, dove $s$ è l'intero sopra considerato e $r=\operatorname{codim}_{Y}\left(Y^{\prime}\right)$.

OSSERVAzIONe 2. - Dna immersione $j: Y^{\prime} \rightarrow Y$ è localmente intersezione completa (cioè $j\left(Y^{\prime}\right)$ è localmente intersezione completa in $X$ ) se e soltanto se l'immersione $j$ è razionale e $i^{*} I=O_{E(P)}(1)$.

EsEmpio 1. - Ogni dilatazione di uno schema $Y$ di centro un sottoschema chiuso $Y^{\prime}$ localmente intersezione completa in $Y$ è razionale; infatti in tali ipotesi è ben noto che il fascio $E$ è il fascio conormale di $Y^{\prime}$ in $Y$ e si ha $i^{*} I=O_{P(R)}(1)$. In particolare ogni dilatazione di uno sehema liscio di centro un sottoschema liscio è una immersione razionale. 
EsEmpio 2. - Sia $Y^{\prime}$ uno schema liscio arbitrario, $E$ un fascio localmente libero su $Y^{\prime}$ di rango $r \geqslant 2$ e $s>0$ un intero; si pone $Y=\operatorname{Spec}\left(S(E)^{(s)}\right)$, e $j: Y^{\prime} \rightarrow Y$ sia l'immersione chiusa associata all'omomorfismo canonico suriettivo

$$
S(E)^{(s)} \rightarrow\left(S(E)^{(s)}\right)_{0}=O_{Y^{\prime}}
$$

In queste ipotesi è ben noto che nel diagramma (*) si ha $X^{\prime}=P(E)$ e $i^{*} I=O_{P(E)}(s)$, vedi [4], ch. II, quindi $j$ è razionale (e questo è il più semplice esempio di immersione razionale).

In particolare, se si prende $Y^{\prime}=\operatorname{Spec}(K)$, con $K$ un corpo algebricamente chiuso, allora $E=K^{r}$ è uno spazio vettoriale di dimensione $r \geqslant 2$ e si ha

$$
Y=\operatorname{Spec} K\left[T_{1}, \ldots, T_{r}\right]^{(s)}=\operatorname{Spec} K\left[\ldots, T_{i_{1}}, \ldots, i_{r}, \ldots\right] / \mathrm{m}
$$

dove $T_{i_{1}, \ldots, i_{r}}$ sono indeterminate tali che $i_{k} \geqslant 0, i_{1}+\ldots+i_{r}=s$ ed $\mathrm{m}$ è l'ideale omogeneo generato dalle forme quadratiche

$$
T_{i_{1}, \ldots, i_{r}} T_{j_{1}, \ldots, j_{r}}-T_{h_{1}, \ldots, h_{r}} T_{t_{2}, \ldots, t_{r}}
$$

con $i_{k}+j_{k}=h_{k}+t_{k}, 1 \leqslant k \leqslant r$, che definiscono il cono affine, con una sola singolarità nell'origine, della varietà di Veronese $V_{r-1}^{s} \subseteq P^{N}(K)$, con $N+1=\left(\begin{array}{c}r+s-1 \\ r-1\end{array}\right)$.

Osservazione 3. - La condizione (ii) della def. 1 si può localizzare. Sia infatti $j: \bar{Y}^{\prime} \rightarrow Y$ una immersione razionale; se $y \in \bar{Y}^{\prime}$, allora indicando con $J_{\nu}$ l'ideale di $Y^{\prime}$ nell'anello locale $O_{x, y}$, esiste un isomorfismo di algebre graduate

$$
\alpha_{y}: \underset{n \geqslant 0}{\oplus} J_{y}^{n} / J_{y}^{n+1} \rightarrow\left(O_{P^{\prime}, y}\left[T_{1}, \ldots, T_{r}\right]\right)^{(s)}
$$

dove $r$ è l'altezza di $J_{y}$.

Definizione 3. - Una coppia $(A, \mathfrak{m})$ formata da un anello locale noetheriano e normale $A$ ed un ideale primo $m$ tale che l'altezza di $\mathfrak{m}(\mathrm{ht}(\mathrm{m}))$ è maggiore o uguale a 2 , è una coppia razionale se l'immersione chiusa $\operatorname{Spec}(A / \mathfrak{m}) \rightarrow \operatorname{Spec}(A)$ è razionale.

Osservazione 4. - Poichè $A / \mathrm{m}$ è un anello locale, Spec $(A / \mathrm{m})$ è uno schema locale. Quindi ogni fascio $E$ localmente libero su $\operatorname{Spec}(A / \mathfrak{m})$ è libero. Ne segue che $(A, \mathfrak{m})$ è una coppia razionale se e soltanto se vi è un isomorfismo di algebre graduate

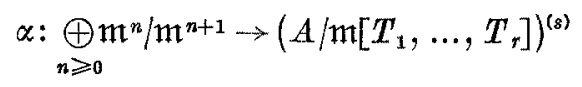

$\operatorname{con} r=h t(\mathfrak{m}) \geq 2$ e $s>0$ (cioè se vale la (ii) della def. 2). 


\section{2. - I teoremi A e B.}

Teorema A. - Siano $(A, \mathfrak{n t})$ una coppia razionale e $f: X \rightarrow Y=\operatorname{Spec}(A)$ la dilatazione di $Y$ di centro $\operatorname{Spec}(A / \mathfrak{H t})$. Allora il gruppo $\mathrm{Pic}(X)$ è ciclico infinito, e, nel caso in cui $i^{*} I=O_{x^{\prime}}(1), \operatorname{Pic}(X) \grave{e}$ generato dalla classe di $O_{x}(1)=I$.

Dimostrazione. - Si procederà con due diverse argomentazioni: la prima con metodi elementari; la seconda, più breve, richiede metodi potenti, ricavati, dal l'exposé XI di [5].

1) Consideriamo il diagramma di dilatazione:

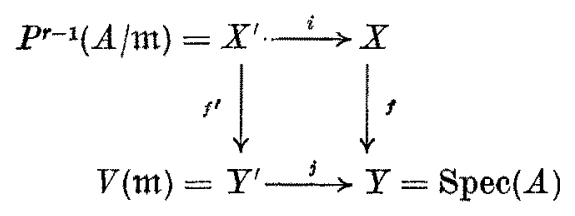

Poichè $A / \mathfrak{m}$ è locale, $\operatorname{Pic}\left(X^{\prime}\right)=Z$ (perchè $\operatorname{Pic}\left(X^{\prime}\right)$ è generato da $O_{X^{\prime}}(1)$ ). Sarà sufficiente allora dimostrare che l'omomorfismo $i^{*}: \operatorname{Pic}(X) \rightarrow \operatorname{Pic}\left(X^{\prime}\right)$ è iniettivo. Infatti, $\operatorname{im}\left(i^{*}\right) \neq 0$, perchè $i^{*} I=O_{x^{\prime}}(s)$. Ora, poichè, se $J$ è l'ideale definito da $\mathfrak{m}$, si ha $X=\operatorname{Proj}\left(O_{Y} \oplus J \oplus J^{2} \oplus \ldots\right)$ ed $f$ è la proiezione $X \rightarrow Y$, si ha per ogni fascio coerente $L$ sopra $X$ (cfr. [4], ch. III):

$$
H^{1}\left(X, L \otimes I^{n}\right)=0 \quad \text { per } n \gg 0 .
$$

Si prende a tal fine per $L$ un fascio invertibile su $X$ tale che $i^{*} L=O_{X^{\prime}}$; si deve dimostrare che $L \cong O_{x}$. Si considera la successione esatta

$$
0 \rightarrow I^{n+1} \rightarrow I^{n} \rightarrow i^{*} O_{X^{\prime}}(n s) \rightarrow 0
$$

(dedotta da $i^{*} I=O_{x^{\prime}}(s), s>0$ ) e mediante tensorizzazione per $L$ (tenendo conto del fatto che $\left.i^{*} I=O_{x^{\prime}}(s)\right)$, si ottiene, per ogni $n \geqslant 0$, la successione esatta

$$
0 \rightarrow L \otimes I^{n+1} \rightarrow L \otimes I^{n} \rightarrow i^{*} O_{x^{\prime}}(n s) \rightarrow 0 .
$$

Ma, poichè $X^{\prime}=P^{r-1}(A / \mathrm{m})$, si ha $H^{1}\left(X^{\prime}, O_{X^{\prime}}(n s)\right)=0$, per $n \gg 0$ (vedi [4], ch. III), da cui si deduce, per induzione decrescente, dalla successione esatta di coomologia

$$
H^{1}\left(X, L \otimes I^{n+1}\right) \rightarrow H^{1}\left(X, L \otimes I^{n}\right) \rightarrow 0
$$

che $H^{1}\left(X, L \otimes I^{n}\right)=0$ per ogni $n \geqslant 0$. 
D'altra parte, scrivendo la successione esatta di coomologia associata a $\left(1_{0}\right)$

$$
H^{0}(X, L) \rightarrow H^{0}\left(X^{\prime}, O_{X^{\prime}}\right) \rightarrow H^{1}(X, L \otimes I)=0,
$$

si ottiene una sezione $\sigma \in H^{0}(X, L)$ tale che $\left.\sigma\right|_{X^{\prime}}=1$, quindi $\sigma(x) \neq 0$ se $x \in X^{\prime}$. Ma l'insieme $X_{\sigma}=\{x \in X \mid \sigma(x) \neq 0\}$ è un aperto contenente $X^{\prime}$, da cui segue $X_{\sigma}=X$ poichè $A$ è locale e per finire $L \cong 0_{x}$.

Se $i^{*} I=O_{x^{\prime}}(1)$ allora $i^{*}$ è suriettivo perchè la classe di $O_{x^{\prime}}(1)$ genera $\operatorname{Pic}\left(X^{\prime}\right)$. Quindi $\operatorname{Pic}(X)=\operatorname{Pic}\left(X^{\prime}\right)$, e questo implica che $\operatorname{Pic}(X)$ è generato dalla classe di $I$.

2) Si può supporre $A$ completo per la topologia mt-adica e in questo caso si può dimostrare che l'omomorfismo $i^{*}: \operatorname{Pic}(X) \rightarrow \operatorname{Pic}\left(X^{\prime}\right)$ è biiettivo; per questo è sufficiente vedere che le due condizioni seguenti (vedi [5], exposé XI, prop. 3.12), sono verificate:

(a) La coppia $\left(X, X^{\prime}\right)$ verifichi la "condizione di Lefschetz effettiva ", cioè il funtore $F \rightarrow \hat{F}$ definito sulla categoria dei fasci coerenti localmente liberi su $X$ a valori nella categoria dei fasci coerenti localmente liberi sul completato formale $\hat{X}$ di $X$ lungo $X^{\prime}$ è una equivalenza di categorie (si tiene conto della circostanza che il solo aperto di $X$ contenente $X^{\prime}$ è lo stesso $X$ ).

(b) $H^{n}\left(X^{\prime}, I^{n} / I^{n+1}\right)=0$, per ogni $n \geqslant 0$ e $q=1,2$.

La prima condizione è conseguenza dei "teoremi di confronto" in Geometria algebrica. Più precisamente, il funtore considerato $F \rightarrow \hat{F}$ è pienamente fedele, poichè, in base al $1^{\circ}$ teorema di confronto (vedi [4], ch. III, (4.1.5)), l'omomorfismo canonico $H^{0}(X, F) \rightarrow H^{0}(\hat{X}, \hat{F})$ è un isomorfismo, tenuto conto del fatto che $H^{0}(X, F)$ è un $A$-modulo di tipo finito (infatti $f$ è un morfismo proprio), quindi completo per la topologia m-adica (essendo $A$ completo). Inoltre per ogni fascio coerente $G$ localmente libero su $\hat{X}$ esiste un fascio coerente $F$ su $X$ tale che $\hat{F}=G$ (in virtù del teorema di esistenza di [4], ch. III, (5.1.4)), il fascio $F$ risulta subito localmente libero su $X$. Nel caso in cui $i^{*} I=O_{x^{\prime}}(1)$ e $A$ non è completo, la classe di $I$ genera $\operatorname{Pic}(X)$ giacchè la stessa proprietà è valevole per la classe di $O_{x^{\prime}}(1)$ in $\operatorname{Pic}\left(X^{\prime}\right)$.

Per finire basta sapere che il solo aperto di $X$ che contiene $X^{\prime}$ è lo stesso $X$. Infatti sia $V=X-F$ un tale aperto, con $F$ chiuso e $F \cap X^{\prime}=\emptyset$; poichè $f$ è proprio $f(X)$ è chiuso in $Y$ e $f(F) \cap Y^{\prime}=\emptyset$. Ne segue che $f(F)=\emptyset$, quindi $F=\emptyset$, cioè $V=X$ poichè $A$ è un anello locale.

CoRollario 1. - Nelle ipotesi del teorema A, si suppone inoltre che l'omomorfismo $h^{*}: \operatorname{Pic}(X) \rightarrow \operatorname{Pic}(U)$ è suriettivo, dove si è posto $U=X-X^{\prime}$ e $h: U \rightarrow X$ è l'immersione aperta corrispondente. Allora $\operatorname{Pic}(U)$ è un gruppo ciclico finito. Se $i^{*} I=O_{x^{\prime}}(1)$, segue che $\operatorname{Pic}(D)=0$ ed $A$ è parafattoriale. Inottre, se l'anello locale $A / \mathfrak{m}$ è regolare, allora $h^{*} \grave{e ̀ ~ s u r i e t t i v o ~ q u a n d o ~} \operatorname{Reg}(X) \grave{e}$ un aperto di $X$, oppure quando $U=Y-Y$ è fattoriale (nel senso che ogni anello locale $O_{v, y}$ con $y \in U$, ̀̀ fattoriale). 
Durostrazione. - Poichè $\operatorname{Pie}(X)$ è ciclico infinito, è sufficiente verificare che $\operatorname{Ker}\left(h^{*}\right) \neq 0$. Ma, evidentemente, $h^{*}(I)=\left.I\right|_{v}=O_{V}$, quindi la classe di $h^{*}(I)$ è $O$, e, d'altra parte, $I \cong O_{x}$ giacchè altrimenti risulterebbe $i^{*} I=O_{x^{\prime}}$, ciò che sarebbe in contraddizione con l'uguaglianza $i^{*} I=O_{x^{\prime}}(s)$, per $s>0$, quindi la classe di $I$ è differente da $O$. Quando $i^{*} I=O_{x^{\prime}}(1)$, abbiamo dunque che l'omomorfismo $h^{*}$ è suriettivo e coincide con l'omomorfismo $O$, quindi $\operatorname{Pic}(U)=0$ e $A$ è parafattoriale, l'altezza di $\mathfrak{a}$ essendo maggiore o uguale a 2 .

Se $A / \mathfrak{m}$ è regolare, allora $X^{\prime}$ è uno schema non singolare e, poichè $I$ è invertibile, $X$ è anch'esso non singolare nei punti di $X^{t}$. Ne segue che $X^{\prime} \subseteq \operatorname{Reg}(X)$ e quindi $\operatorname{Reg}(X)=X$, perchè $\operatorname{Reg}(X)$ è aperto. Se $\operatorname{Reg}(X)$ non è aperto, ma $U$ è uno schema fattoriale, ci viene dimostrato che $X$ è uno schema fattoriale. In ogni caso, la suriettività di $h^{*}$ risulta subito, tenendo conto del fatto che il gruppo $\operatorname{Pic}(X)$, rispettivamente il gruppo $\operatorname{Pic}(U)$, s'identifica con il gruppo delle classi dei divisori di Weil su $X$, rispettivamente su $U$.

CoRollario 2. - Nelle ipotesi del corollario 1, sia $A$ un anello noetheriano e normale e $\mathfrak{m} \subseteq A$ un ideale primo di altezza $\geqslant 2$ tale che $A / \mathfrak{m}$ sia regolare. La coppia $(A, \mathfrak{m})$ verifichi le condizioni del teorema $A$ e supponiamo inoltre che $U=X-Y^{\prime}$ sia fattoriale. Allora il gruppo $\operatorname{Pic}(D(\mathfrak{m}))$ è finito da cui discende che $A$ è semifattoriale (cioè il gruppo delle classi dei divisori di $A$ è di torsione, ofr. [9]). Infine, se $i^{*} I=O_{x^{\prime}}(1)$, allora è fattoriale, oppure se $i^{*} I=O_{x^{\prime}}(s)$ con $s>0$, ma $A$ ̀े completo nella topologia m-adica, allora il gruppo delle classi dei divisori di $A$ è isomorfo con il gruppo ciclico finito ai ordine $s, Z / s Z$.

Dimostrazione. - Osserviamo che $D(\mathfrak{n t})=Y-Y^{\prime}=X-X^{\prime}=U$ e, applicando il corollario 1 , si ha che $\operatorname{Pic}(D(\mathfrak{m}))$ è finito. Poichè $h t(\mathfrak{m}) \geqslant 2$, ne segue che l'applicazione $\operatorname{Pic}(U) \rightarrow \mathrm{Cl}(A)$ è biiettiva (quì $\mathrm{Cl}(A)$ indica il gruppo delle elassi dei divisori di $A$ ), quindi $A$ è semifattoriale (nel caso generale dove $i^{*} I=O_{x^{\prime}}(s) s>0$ ) e fattoriale se $s=1$.

Infine supponiamo $A$ completo per la topologia m-adica. Allora la seconda dimostrazione del teorema $A$ implica che $\operatorname{Pic}(X) \cong \operatorname{Pic}\left(X^{\prime}\right)$. Per concludere basta sapere che $\operatorname{Ker}\left(h^{*}\right)$ è il sottogruppo di $\operatorname{Pic}(X)$ generato dalla classe di $I$ (che corrisponde alla classe di $O_{z^{\prime}}(s)$ in $\left.\operatorname{Pie}\left(X^{\prime}\right)\right)$. $X$ essendo uno sehema fattoriale (anche regolare nei punti di $X^{\prime}$ poichè l'anello $A / \mathfrak{m}$ è regolare), ne segue che il gruppo $\operatorname{Pic}(X)(\operatorname{risp}$. il gruppo $\operatorname{Pic}(U))$ coincide con il gruppo delle classi dei divisori di Weil su $X$ (risp. su $U$ ).

Sia allora $L$ un fascio invertibile su $X$ associato al divisore di Weil $D=\sum_{i=1}^{\alpha} n_{i} D_{i}+n X^{\prime}$ con $D_{i} \neq X^{\prime}$, tale che $\left.L\right|_{U}=O_{v}$, cioè tale che $\left.D\right|_{U}=\left.\sum_{i=1}^{\alpha} n_{i} D_{i}\right|_{U}$ è principale. Esiste allora una funzione razionale $f$ (su $U$ ) tale che il divisore di $f$ su $U$, $\operatorname{div}_{\sigma}(f)$, sia uguale a $\left.D\right|_{D}$. Da ciò discende che il divisore di $f$ su $X, \operatorname{div}_{X}(f)$, è uguale a $\sum_{i=1}^{\infty} n_{i} D_{i}+m X^{\prime}\left(n_{i}, n, m\right.$ sono interi) e finalmente $D \sim(n-m) X^{\prime}$ (equivalenza lineare), cioè $L=\left(I^{-1}\right)^{n-m}$ (perchè $I^{-1}$ è il fascio invertibile associato al divisore $\left.X^{\prime}\right)$. Quindi $\operatorname{Ker}\left(h^{*}\right)$ è contenuto 
nel sottogruppo di $\operatorname{Pic}(X)$ generato dalla classe di $I$. Poichè l'opposta inclusione è immediata, la dimostrazione è finita.

Osservazione 5. - Dal corollario 2 si può dedurre che ogni anello locale noetheriano e normale $A$, con $\mathfrak{n}$ l'ideale massimale tale che $U=\operatorname{Spec}(A)-\mathfrak{n}$ è fattoriale, avente la proprietà che esista un isomorfismo di algebre graduate:

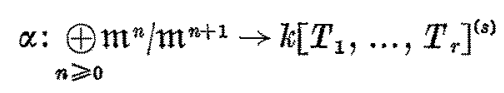

(con $k=A / \mathrm{m}$ il campo residuo, le $T_{i}$ variabili) è semifattoriale. In particolare si ritrova come applicazione che l'anello locale del vertice del cono affine della varietà di Veronese è semifattoriale, da cui segue, applicando la prop. 7.4 di [7], il coroll. 1 di [3], affermante che la varietà di Veronese, immagine proiettiva del sistema lineare delle ipersuperficie di ordine $s$ di $P^{r-1}(k)$, con $k$ un campo algebricamente chiuso, è aritmeticamente semifattoriale.

Possiamo introdurre a questo punto la seguente:

Definizione 4. - Sia $A$ un anello locale di dimensione $r \geqslant 2$, d'ideale massimale $\mathfrak{n t}$ e di campo residuo $k$. L'anello $A$ dicesi semiregolare di tipo $s(s>0)$, se

(i) la coppia $(A, \mathfrak{m})$ è razionale e, più precisamente, abbiamo che il graduato di $A$ rispetto a $\mathfrak{m}$ è isomorfo con $k\left[T_{1}, \ldots, T_{q}\right]^{(s)}$.

(ii) l'insieme $U=\operatorname{Spec}(A)-\mathfrak{m}$ è regolare.

È chiaro che un anello semiregolare di tipo 1 è regolare.

Osservazione 6. - Se l'anello $A$ è semiregolare di tipo $s$ e di dimensione $r$, allora considerando il diagramma di dilatazione di $Y=\operatorname{Spec}(A)$ lungo $Y^{\prime}=\operatorname{Spec}(k)$ (vedi def. 2), abbiamo che $X^{\prime}=P^{r-1}(k)$ e $i^{*} I=O_{X^{\prime}}(s)(s>0)$.

Si verifica facilmente che se $r>1$, la coppia $(A, \mathfrak{m})$ verifica le ipotesi del coroll. 2, quindi:

(1) ogni anello semiregolare è semifattoriale;

(2) se $A$ è un anello semiregolare, $\hat{A}$ (il completato di $A$ nella topologia m-adica) è fattoriale se e solo se è $A$ regolare.

La (1) è immediata.

La (2) discende dal fatto che $\hat{A}$ è fattoriale se e solo se $s=1$, poichè $\mathrm{Cl}(\hat{A})=Z / s Z$ (coroll. 2).

EsEmpio 3. - L'anello locale del vertice del cono affine della varietà di Veronese è semiregolare. 
Il corollario seguente ha analogia, in caratteristica arbitraria, con un teorema di Grauent (Mathm. Annalen-146 (1962), pp. 331-368, $\$ 4$ teor. 7) per gli spazi complessi, ma il metodo utilizzato è del tutto differente.

CoRollario 3. - Siano $A$ e $A^{\prime}$ due anelli locali semiregolari di dimensione $r \geqslant 2$ e di tipo $s>0$ aventi lo stesso corpo residuo to tali ohe $A$ e $A^{\prime}$ contengano il corpo $k$. Allora $i$ completati $\hat{A}$ e $\hat{A}^{\prime} d i$ A e risp. di $A^{\prime}$ nelle topologie degli ideali massimali sono isomoriti.

Dimostrazione. - È sufficiente dimostrare che, se $A$ è un anello locale completo semiregolare contenente il suo corpo residuo, allora $A$ è isomorfo al completato dell'anello locale del vertice del cono affine della varietà di Veronese (cfr. esempio 3).

Nel considerare il diagramma della dilatazione dì $Y=\operatorname{Spec}(A)$ di centro $Y^{\prime}=\operatorname{Spec}(k)=\mathfrak{n}$

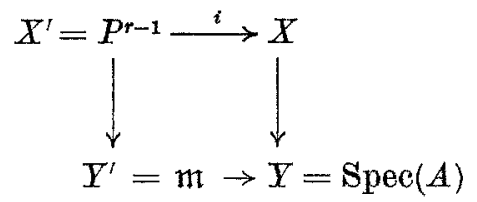

si ̀̀ visto nella seconda dimostrazione del teorema $A$ che l'omomorfismo $i^{*}: \operatorname{Pic}(X) \rightarrow \operatorname{Pic}\left(X^{\prime}\right)$ è un isomorfismo. Esiste quinđi un fascio invertibile $L$ su $X$ tale che $i^{*} L=O_{x^{\prime}}(1)$ e denotiamo con $S$ l'algebra graduata $\oplus H^{0}\left(X, L^{\otimes n}\right)$.

Si ha $H^{1}(X, L \otimes I)=0$; infatti, così come nella prima dimostrazione del teorema $A$, si ha che $H^{1}\left(X, I \otimes I^{n}\right)=0$ per $n \gg 0$ e dalla successione esatta:

$$
0 \rightarrow L \otimes I^{n+1} \rightarrow L \otimes I^{n} \rightarrow i^{*} o_{x^{\prime}}(n s+1) \rightarrow 0
$$

se ne deduce per induzione decrescente che $H^{1}\left(X, I \otimes I^{n}\right)=0$ per ogni $n \geqslant 0$, da cui l'affermazione. La successione esatta di coomologia associata alla successione esatta precedente per $n=0$

$$
H^{0}(X, L) \rightarrow H^{0}\left(X^{\prime}, O_{X^{\prime}}(1)\right) \rightarrow H^{1}(X, L \otimes I)=0
$$

mostra che vi sono $r=\operatorname{dim}(A)$ sezioni $\varepsilon_{1}, \ldots, \varepsilon_{r} \in H^{0}(X, L)$ tali che le restrizioni a $X^{\prime}$ siano le variabili $T_{1}, \ldots, T_{r}$ (l'algebra graduata $\oplus H^{\prime}\left(X^{\prime}, O_{X^{\prime}}(n)\right)$ è canonicamente isomorfa all'algebra di polinomi $\left.k\left[T_{1}, \ldots, T_{r}\right]\right)$.

Siano inoltre $a_{i_{1}, \ldots, i_{r}} \in H^{0}\left(X, L^{\otimes s}\right)=H^{0}(X, I)$ (infatti, si ha $L^{\otimes s}=I$, giacchè i due fasci hanno la stessa immagine in $\operatorname{Pic}\left(X^{\prime}\right)$ e cioè $\left.O_{X^{\prime}}(s)\right)$ per $i_{k} \geqslant 0$ e $i_{1}+\ldots+i_{r}=s$ le sezioni seguenti

$$
a_{i_{1}, \ldots, i_{r}}=\varepsilon_{1}^{i_{1}} \ldots \varepsilon_{r}^{i_{r}}
$$

il prodotto degli $\varepsilon_{i}$ essendo preso nell'algebra graduata $S$. È evidente che $H^{\circ}(X, I)=\mathrm{m}$ (giacchè $\mathfrak{m}$ è massimale, ovvero in virtù del teor. 1 di [1]) e d'altra parte questi ele- 
menti di m soddisfano chiaramente le equazioni di Veronese:

$$
a_{i_{1}, \ldots, i_{r}} a_{i_{1}, \ldots, j_{r}}-a_{h_{1}, \ldots, h_{r}} a_{t_{1}, \ldots, t_{r}}=0\left(i_{k}+j=h_{k}+t_{k}, i \leqslant k \leqslant r\right)
$$

D'altra parte, la successione esatta

$$
0 \rightarrow I^{2} \rightarrow I \rightarrow i^{*} O_{x^{\prime}}(s) \rightarrow 0
$$

conduce alla successione esatta di coomologia

$$
0 \rightarrow H^{0}\left(X, I^{2}\right)=\mathfrak{m}^{2} \rightarrow H^{0}(X, I)=\mathfrak{m} \rightarrow H^{0}\left(X^{\prime}, O_{x^{\prime}}(s)\right) \rightarrow H^{1}\left(X, I^{2}\right)=0
$$

e, poichè tutti i monomi di grado $s$ in $T_{1}^{\prime}, \ldots, T_{r}$ formano una base per il $k$-spazio vettoriale $H^{0}\left(X^{\prime}, o_{x^{\prime}}(s)\right)$, ne risulta che gli $a_{i_{1}, \ldots, i_{r}}\left(\right.$ in numero di $\left.\left(\begin{array}{c}r+s-1 \\ r-1\end{array}\right)\right)$ generano l'ideale $\mathfrak{n t}$ (utilizzando il lemma di Nakayama).

Poichè il corpo $k$ è contenuto in $A$, si ha un omomorfismo unico di $k$-algebre:

$$
k\left[\ldots T_{i_{1}, \ldots, i_{r}} \ldots\right] \rightarrow A
$$

che manda le variabili $T_{i_{1}, \ldots, i_{r}}$ nelle $a_{i_{1}, \ldots, i_{r}}$, giacchè le $a_{i_{1}, \ldots, i_{r}}$ soddisfano le relazioni $(* *)$, questo omomorfismo induce un omomorfismo unico

$$
B=k\left[\ldots T_{i_{1}, \ldots, i_{r}} \ldots\right] / \mathfrak{b} \rightarrow A
$$

dove $\mathfrak{b}$ è l'ideale omogeneo generato dalle equazioni della varietà di Veronese (analoghe alle $(* *)$ ), da cui un omomorfismo unico $A^{\prime} \rightarrow A$ di $k$-algebre con $A^{\prime}$ il localizzato di $B$ nell'ideale massimale corrispondente all'origine (vertice del cono di Veronese). Ma essendo $A$ completo per la topologia m-adica, si ottiene un omomorfismo unico di $k$-algebre $\hat{A}^{\prime} \rightarrow A$ con $\hat{A}^{\prime}$ il completato di $A^{\prime}$ per la topologia m'-adica ( $\mathfrak{m}^{\prime}$ essendo l'ideale massimale di $A^{\prime}$ che è generato dalle classi delle $\left.T_{i_{2}, \ldots, i_{r}}\right)$. Questi due anelli sono integri, hanno la stessa dimensione, $r$, e $\hat{\mathfrak{m}}^{\prime} A=\mathfrak{m}$ (per costruzione dell'anello $A^{\prime}$ ). Per il lemma di Nakayama risulta che questo omomorfismo è suriettivo, quindi anche biiettivo a causa delle dimensioni.

CORoLlARIo 4. - Un anello semiregolare contenente il suo corpo residuo è sempre un anello di Cohen-Macaulay.

Dimostrazione. - Per il corollario 3 , è sufficiente (giacchè un anello locale noetheriano è di Cohen-Macaulay se e soltanto se lo è il suo completato) verificare che l'anello locale del vertice del cono affine della varietà di Ver onese è un anello di Cohen-Macaulay e questo è un fatto ben noto. D'altra parte ciò si dimostra anche facilmente osservando che i monomi $T_{1}^{s}, \ldots, T_{r}^{s}$ formano una successione regolare nell'anello (di dimensione di Krull $r$ ) $k\left[T_{1}, \ldots, T_{r}\right]^{(s)}$. 
Osservazione 7. - Ci si può chiedere se un anello semiregolare è oppure non è di Gorenstein. Il coroll. 3 riduce il problema (per gli anelli che contengono il loro corpo residuo) al caso del localizzato nell'origine dell'anello $k\left[T_{1}, \ldots, T_{r}\right]^{(s)}$; ora, in questo caso si possono compiere dei calcoli elementari espliciti per vedere ciò. La risposta è la seguente: è un anello di Gorenstein se e soltanto se $s$ divide $r$. Sotto questa forma questo risultato è stato ottenuto da $V$. BRînZăNESOU (vedi Studii Cerc. Metematicè, Tom. 26, Nr. 5(1974) pp. 625-629), mentre, per $r=2$, è stato ottenuto da M. Frorentini in [3].

TEorema B. - Siano $A$ un anello locale noetheriano di dimensione $r \geqslant 2$, d'ideale massimale $\mathfrak{m}$ e di campo residuo $k$, e sia

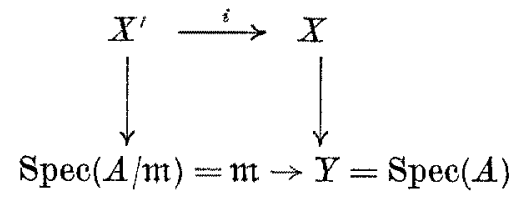

il diagramma cartesiano dello scoppiamento di $Y$ di centro $\operatorname{Spec}(A / \mathfrak{m})$; si supporrà che $X^{\prime}$ sia una varietì non singolare ammettente una immersione $h: X^{\prime} \rightarrow P^{n}(k)$ tale che, se $O_{X^{\prime}}(1)=h^{*} O_{p^{n}(\{k)}(1)$, allora il gruppo $\mathrm{Pic}\left(X^{\prime}\right)$ sia generato dalla classe $d i O_{X^{\prime}}(1)$ e che $X^{\prime}$ sia 2-volte di prima specie relativamente ad $h$ (vedi [8], ch. III). In queste condizioni $\operatorname{Pic}(X)$ è un gruppo cictico infinito. Se $\operatorname{Reg}(X) \grave{e}$ aperto in $X$, oppure se $X-X^{\prime}$ $\grave{e}$ fattoriale $A$ è semifattoriale. Se, inoltre, $i^{*} I=O_{x^{\prime}}(1)$, allora A è fattoriale.

Dmostraztone. - Si può osservare che $i^{*} I$ è ampio su $X^{\prime}$, quindi $i^{*} I=O_{X^{\prime}}(s)$ con $s>0$ e $H^{1}\left(X^{\prime}, O_{X^{\prime}}(m)\right)=0$ per ogni $m \in Z$, poichè $X^{\prime}$ è 2-volte di prima specie relativamente ad $h$. Inoltre $X$ è non singolare in ogni punto di $X^{\prime}$, poichè lo è $X^{\prime}$ e l'ideale $I$ è invertibile. Si può ora ripetere punto per punto la dimostrazione del teorema $A$, mostrando che l'omomorfismo $i^{*}: \operatorname{Pic}(X) \rightarrow \operatorname{Pic}\left(X^{\prime}\right)$ è iniettivo. Quindi $\operatorname{Pic}(X)$ è ciclico infinito. La semifattorialità di $A$ si dimostra con lo stesso procedimento utilizzato nella dimostrazione del coroll. 1 . Se inoltre $i^{*} I=O_{x^{\prime}}(1)$, allora $i^{*}$ è anche biiettivo, giacchè $O_{X^{\prime}}(1)$ genera $\operatorname{Pic}\left(X^{\prime}\right)$, quindi $I$ genera $\operatorname{Pic}(X)$. Se ne deduce che $\operatorname{Pic}(Y-\mathfrak{m})=0$, da cui $\mathrm{Cl}(A)=0$, come nella dimostrazione del coroll. 2, cioè $A$ è fattoriale.

Osservazione 8. - Nelle ipotesi del teorema $B$, se inoltre $X^{\prime}$ è 3-volte di prima specie si può dimostrare che $\mathrm{CI}(\hat{A})=Z / s Z$, dove $i^{*} I=O_{X^{\prime}}(s)$ con $s>0$.

\section{3. - Applicazioni geometriche dei teoremi $\mathrm{A}$ e $\mathrm{B}$.}

Come applicazioni geometriche dei teoremi A e B, si ottengono i seguenti teoremi $\mathrm{A}$ bis e $\mathrm{B}$ bis, che forniscono ulteriori informazioni sulle contrazioni in legame con i risultati stabiliti, rispettivamente, col teorema 2 di [1], nonchè col teorema 1 di [2] per le varietà algebriche irriducibili sopra un campo $k$ algebricamente chiuso. 
Teorema A bis. - Sia

$(*)$

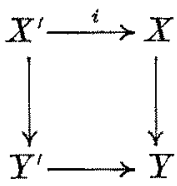

un diagramma di contrazione razionale di varietà algebriche sopra $k$, cioè un diagramma tale che:

1) $X^{\prime}=P(E)$, con $E$ fascio localmente libero di rango $r \geqslant 2$ su $\bar{Y}^{\prime}$ e $P(E)$ il fibrato proiettioo associato ad $E$;

2) $i^{*} I=O_{P(B)}(s)$ con $s>0$, dove $I$ è l'ideale di $X^{\prime}$ in $O_{x}$. Inoltre $Y$ è normale in ogni punto di $Y^{\prime}$. Si suppone che $Y^{\prime}$ è non singolare. Si ha ehe:

(i) per ogni $y \in Y^{\prime}$, l'anello locale $O_{Y, y}$ è semifattoriale;

(ii) $s e i^{*} I=O_{P(E)}(1)$, allora $O_{Y, y}$ è regolare.

Drmostrazione. - Sia $J \subseteq O_{Y}$ l'ideale di $Y^{\prime}$ e $y \in Y^{\prime}$. Sia $\operatorname{Spec}\left(O_{Y, y}\right) \rightarrow Y$ il morfismo canonico definito dall'applicazione identica $O_{Y, y} \rightarrow O_{Y, y}$ (cfr. [4], ch. I). Dal diagramma cartesiano $(*)$ con cambiamento di base, mediante $\operatorname{Spec}\left(O_{Y, y}\right) \rightarrow Y$ si ottiene il diagramma cartesiano

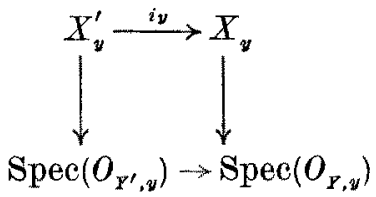

dove $X_{y}^{\prime}=\operatorname{Proj}\left(O_{Y^{\prime}, y}\left[T_{1}, \ldots, T_{r}\right]\right)$, perchè $X^{\prime}=P(E)$, con $E$ modulo su $O_{Y^{\prime}}$, localmente libero di rango $r$; inoltre $X_{y} \rightarrow \operatorname{Spec}\left(O_{r, y}\right)$ è la dilatazione di $\operatorname{Spec}\left(O_{r, v}\right)$ lungo Spec $\left(O_{x^{\prime}, y}\right)$. Si osservi che se $I_{y} \subseteq O_{x_{y}}$ è l'ideale di $X_{y}^{\prime}$, dalla condizione $i^{*} I=O_{X^{\prime}}(s)$ nel diagramma (*), si ricava $i_{y}^{*} I_{y}=O_{x^{\prime}}(s)$ con $s>0$. Quindi se si prende $A=O_{Y, y}$ e $\mathfrak{m}=I_{y}$, la coppia $(A, \mathfrak{m})$ è una coppia razionale. D'altra parte, $Y^{\prime}$ regolare implica $O_{Y^{\prime}, y}$ regolare e quindi anche $X_{y}^{\prime}$ è regolare. Ora $X^{\prime}$ è regolare perchè $I$ è invertibile. Questo fatto implica $X_{y}$ regolare in un intorno aperto di $X_{y}^{\prime}$ il quale coincide con tutto $X_{y}$ (si ricordi l'ultima parte della dimostrazione del coroll. 1). Ne segue che le ipotesi del teorema $A$ e dei corollari 1 e 2 sono soddisfatte. Così si è verificato che $O_{Y, y}$ è semifattoriale. La (ii) è già stata dimostrata in [1].

Teorema B bis. - Sia

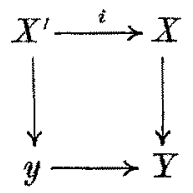


il diagramma di dilatazione di $Y$ nel punto chiuso y. Si suppone che $\operatorname{dim}\left(X^{\prime}\right) \geqslant 3$, $X^{\prime}$ è non singolare ed inoltre esiste una immersione chiusa $X^{\prime} \rightarrow P^{n}(k)$ tale che $X^{\prime}$ diventa una intersesione completa in $P^{n}(k) ; O_{X^{\prime}}(1)$ indica il fascio invertibile definito da $O_{p^{n}}(1)$ mediante l'immersione $X^{\prime} \rightarrow P^{n}(k)$. Sotto queste ipotesi, abbiamo che $y$ è una singolarità isolata di $Y$ e

(j) $O_{Y, y}$ è semifattoriale;

(jj) se $i^{*} I=O_{X^{\prime}}(1)$, allora $O_{\boldsymbol{r}, y} \grave{e}$ fattoriale.

Dumostraztone. - Con un procedimento analogo a quello seguito nella precedente dimostrazione, si applica il teorema $B$, tenendo conto dei fatti seguenti: poichè $i^{*} I$ è ampio ne segue $i^{*} I=O_{x^{\prime}}(s), s>0$, una intersezione completa di dimensione $\geqslant 3$ è sempre 3-volte di prima specie (vedi [8], ch. III) e, per finire, Pic $\left(X^{\prime}\right)$ è il gruppo libero generato dalla classe del fascio $O_{\mathbf{X}^{\prime}}(1)$ (cfr. [5], exposé XII, coroll. 3.7).

\section{BIBLIOGRAFIA}

[1] L. BADEscu, Contractions rationnelles des variétés algébriques, Ann. Sc. Normale Sup. Pisa, Vol. XXVII (1973), pp. 743-767.

[2] L. Badescu - M. Moroianu, Algebraic contractions and complete intersections, Rend. Acc. Lincei, vol. LII, fasc. 6 (1972), pp. 884-892.

[3] M. Fronentint, Esempi di anelli di Cohen Macaulay semifattoriali che non sono di Gorenstein, Rend. Ace. Lincei, fasc. 5 (1971).

[4] A. Grotmendieck - J. Dievdonné, E.G.A., IHES, ch. I, II, III.

[5] A. GRoTHENDIEck, Séminaire de Géométrie Algébrique, II, 1962.

[6] A. LAsco, Sous-variétés régulierement contractibles d'une variété algébrique, Ann. Se. Normale Sup. Pisa, fasc. 4 (1969).

[7] P. Samuex, Unique factorisation domains, Tata Institute, 1964.

[8] J. P. SkRRr, Faisceawx algébriques cohérentes, Annals of Math., 1955.

[9] U. SToRch, Fastfaktorielle Ringe, Math. Univ. Munster, 1967. 